\title{
Les jardins urbains de Séville
}

L'engagement citadin face au désintérêt de l'Administration

Los huertos urbanos de Sevilla : el compromiso ciudadano frente al desinterés de la Administración

The urban gardens of Seville: the civil commitment against the indifference of the administration

\section{Raúl Puente Asuero}

Traducteur : Dominique Fournier

\section{OpenEdition} Journals

Édition électronique

URL : http://journals.openedition.org/ethnoecologie/2317

DOI : 10.4000/ethnoecologie.2317

ISSN : 2267-2419

Éditeur

Laboratoire Eco-anthropologie et Ethnobiologie

\section{Référence électronique}

Raúl Puente Asuero, «Les jardins urbains de Séville », Revue d'ethnoécologie [En ligne], 8 | 2015, mis en ligne le 31 décembre 2015, consulté le 19 avril 2019. URL : http://journals.openedition.org/ ethnoecologie/2317; DOI : 10.4000/ethnoecologie.2317

Ce document a été généré automatiquement le 19 avril 2019

\section{c) (†)}

Revue d'ethnoécologie est mis à disposition selon les termes de la licence Creative Commons Attribution - Pas d'Utilisation Commerciale - Pas de Modification 4.0 International. 


\title{
Les jardins urbains de Séville
}

\author{
L'engagement citadin face au désintérêt de l'Administration
}

Los huertos urbanos de Sevilla : el compromiso ciudadano frente al desinterés de la Administración

The urban gardens of Seville: the civil commitment against the indifference of the administration

\section{Raúl Puente Asuero}

Traduction : Dominique Fournier

\section{Introduction}

1 L'agriculture urbaine est tout sauf une nouveauté en Espagne, comme en témoignent les riches patrimoines agricole, culturel et gastronomique qui lui sont associés dans toutes les régions du pays. Pourtant, dans le contexte actuel de crises à répétition et comme une réponse élaborée par la population, le nombre des jardins familiaux et urbains s'accroît aujourd'hui de façon exponentielle.

2 Alors qu'elle avait été perçue dans un premier temps comme une crise financière provoquée par l'éclatement de la bulle immobilière, la crise espagnole (2008-....) a entraîné une crise sociale sans précédent (chômage, expulsions, émigration) associée à une crise territoriale et environnementale (bétonnage du littoral, régression des plages, disparition des zones humides) dont on ne perçoit toujours pas la fin. En réponse, se déploie une puissante dynamique agri-urbaine qui s'efforce de contenir ces effets dévastateurs du boom immobilier. Les jardins qui en sont issus s'affirment ainsi aujourd'hui comme les garants d'indépendance et de sécurité alimentaires ainsi que, plus largement, comme un recours social pour les familles défavorisées.

3 Nous proposons ici une illustration de cette dynamique à partir de l'analyse de l'évolution des jardins potagers à Séville depuis les années 90 jusqu'à aujourd'hui. Nous mettrons en lumière les causes et les facteurs qui expliquent cette évolution et soulignerons en particulier le rôle fondamental qu'ont joué les associations de voisins dans cette évolution. 


\section{Le contexte historique}

4 Les jardins internes et extérieurs à la cité existent depuis le néolithique, et nous disposons tout au long de l'histoire de Séville d'un grand nombre d'exemples de jardins et de potagers à fonctions multiples ayant servi, entre autres, à l'approvisionnement alimentaire des habitants.

5 Dès l'époque romaine, on trouvait ainsi déjà à Séville des domus ou maisons qui s'organisaient autour d'un patio central équipé de réserves d'eau, de plantes médicinales et d'arbres fruitiers. Ces jardins avaient une double fonction, puisqu'on s'en servait autant comme espaces de travail que comme lieux de repos. On connaît le goût de l'aristocratie romaine pour l'horticulture, au point que «selon Champlin, l'horticulture constituait le deuxième passe-temps des Romains après la culture » (Rodríguez López 2008). À cette époque déjà, les potagers et les jardins d'ornement se confondaient et se mélangeaient, rassemblant autant de plantes ornementales qu'alimentaires, au point que le mot hortus finit par désigner indistinctement les jardins, les potagers ou les vergers. L'époque musulmane qui suivit a, quant à elle, favorisé la création des plus importants des jardins de la ville. Ceux-ci restaient cependant fondés sur le même modèle qu'à l'époque romaine : des espaces mixtes où jardins d'ornement et potagers se mélangent et se confondent.

6 L'existence de ces nombreux jardins intra et extra-muros répartis sur l'ensemble de la ville continue à être attestée jusqu'au XII siècle, par les documents rédigés à l'occasion de la reconquête de Séville en 1248 par le roi Fernando III et la «répartition » des biens confisqués aux musulmans qui s'ensuivit. Et ce n'est qu'aux $\mathrm{XVI}^{\mathrm{e}}$ et $\mathrm{xVII}^{\mathrm{e}}$ siècles qu'apparaissent clairement les premières traces de leur recul. Ils commencent alors à souffrir de l'accroissement sensible de la population et du développement corrélé de l'urbanisme, tous deux favorisés par l'émergence du commerce avec l'Amérique. Ce développement a lieu à la fois intra-muros, avec l'occupation des petits jardins et potagers, et à l'extérieur de la muraille, avec la mise en place de quelques-uns des faubourgs les plus notables de la ville, tels la Macarena dans le secteur nord, San Bernardo à l'est ou Triana au sud-ouest.

7 Ce démembrement progressif des jardins et des extensions maraîchères s'accentuera à la fin $d u X{ }^{2}{ }^{e}$. Leur superficie va ainsi progressivement s'amenuiser au rythme des besoins de la ville en matière de terrains destinés aux installations et aux infrastructures de base (canalisations de l'eau, voies urbaines, cimetières, édifices religieux, etc.). Ces dernières vont continuer à s'accroitre au-delà de l'enceinte de la ville à partir du xix siècle, provoquant une première modification radicale d'un paysage qui jusqu'ici restait profondément rural. En se portant sur la proche ceinture (extérieure) des murailles, l'expansion de la ville génère une toute nouvelle cohabitation entre des potagers et des fermes agricoles d'une part, et des équipements publics (cimetières, hôpitaux psychiatriques, industries) désormais relégués hors de la cité « historique », d'autre part.

8 Mais ce sont les années 1950 et 1960 qui sont probablement à l'origine des transformations les plus radicales de la morphologie de Séville. Le développement urbanistique de la ville parvient en effet à des niveaux jamais atteints jusqu'alors, dans les zones nord, est et sud. Des entreprises de construction investissent des parcelles de jardins existants dans de vastes zones de la ville pour y bâtir des quartiers de façon 
accélérée, avec l'intention d'y loger un maximum de gens dans le minimum d'espace possible. Il se produit ainsi une croissance par sauts spatiaux, transformant les ensembles d'immeubles en autant de «paquets résidentiels » isolés les uns des autres, et sans aucune communication directe entre eux. Ce développement urbain en "confetti», pour reprendre l'expression de Marie-Elisabeth Chassagne (1982), provoque la disparition rapide des espaces de jardins et potagers. Le paysage antérieur se voit remplacé par un véritable "kaléidoscope » de quartiers qui conservent néanmoins, en de nombreuses occurrences, la structure physique et le nom des jardins disparus: Huerta de la Salud, Huerta del Hierro, Huerta del Rey, Huerta del Peligro, Huerta del Pilar, Huerta del Sol, Huerta de la Papachina, Huerta de San Jacinto, Huerta de Santa Teresa, etc. Une telle croissance non planifiée provoque en outre une série de problèmes auxquels allaient être confrontés les nouveaux habitants de ces quartiers dont, notamment, le manque d'équipements et de services publics (collèges, centres de santé, espaces verts, etc.), l'altération du patrimoine historique artistique, naturel et culturel ainsi qu'une absence de repères identitaires.

Au cours de la dictature (1939-1975), les associations de voisins ayant été interdites, les habitants se trouvèrent dans l'incapacité de revendiquer ou d'intervenir dans les affaires de la ville ou de leurs quartiers. Il fallut attendre l'arrivée de la démocratie au moment de la Transition, et avec elle une législation propre aux comités de quartiers, pour voir des citoyens s'impliquer très activement dans la quête d'une amélioration de leur qualité de vie. Les habitants des quartiers de la périphérie de Séville exprimèrent ainsi leur volonté de jouir des mêmes services et équipements publics que les Sévillans des zones plus favorisées. C'est dans ce but qu'apparurent bientôt des associations dans les quartiers périphériques (Polygone nord, le Polygone San Pablo, le Parc Alcosa, Pino Montano, Torreblanca, etc.).

\section{Le cas du Parc Miraflores}

10 Le premier collectif de jardin horticole urbain de Séville, l'association Comité Pro-Parque Educativo Miraflores, naît en 1983 de cette ferveur associative. Constitué à l'origine par des membres des associations de parents d'élèves (AMPAs), ce groupement était, et il est toujours, en étroite relation avec le monde de la formation et de l'enseignement. L'association se caractérise donc depuis ses débuts par la militance vicinale, sociale, politique et syndicale de ses créateurs (García et al. 2007)

11 Au-delà des avancées sociales qu'ils commençaient à obtenir pour la zone nord de la ville, certains habitants ne se contentèrent plus de réclamer des équipements et des services, ils revendiquèrent une autre manière de penser et de construire la cité, ainsi qu'une alternative au système éducatif institutionnel. À l'époque et encore aujourd'hui, il était, selon eux, indispensable de soutenir le travail d'éducation formelle en vigueur dans les collèges et instituts, par des initiatives d'éducation informelle qui seraient proposées dans le voisinage immédiat. Les membres du Comité Pro-Parque, intégrés dans diverses associations de parents d'élèves, mûrissaient ainsi l'idée que l'éducation en vigueur dans les collèges de la zone ne répondait pas vraiment à leur mission de formation et qu'elle condamnait à terme de nombreux jeunes à venir grossir les poches de marginalité et de sous-développement qui caractérisaient un certain nombre de ces espaces urbains. Il ne suffisait pas à leurs yeux d'améliorer la qualité de l'enseignement au sein des collèges, il fallait aussi réformer l'environnement éducatif, travailler au niveau du quartier, dans les 
familles, avec des collectifs d'adultes, des groupements de jeunes, etc. (Bejarano et al. 2000).

12 C'est sur cette base qu'il fut décidé d' okuper $^{1}$ » et de convertir le Parc de Miraflores, transformé en immense dépotoir avant les années 1980, en un espace où l'on pourrait pratiquer l'éducation informelle en recourant à toutes les ressources qu'il pouvait offrir (jardins, vestiges archéologiques, etc.). Le processus atteint son apogée en 1991 lorsque les jardins scolaires commencèrent à fonctionner, puis en 1992 avec la constitution des jardins d'agrément. Au cours des premières années, tout ceci se développa de manière informelle, voire illégale, jusqu'à ce qu'en 1994, la mairie de Séville, consciente du succès social de l'initiative, se décide à signer une convention avec le Comité Pro-Parque.

Figure 1 : Marche de protestation à travers le Parc de Miraflores (1990)

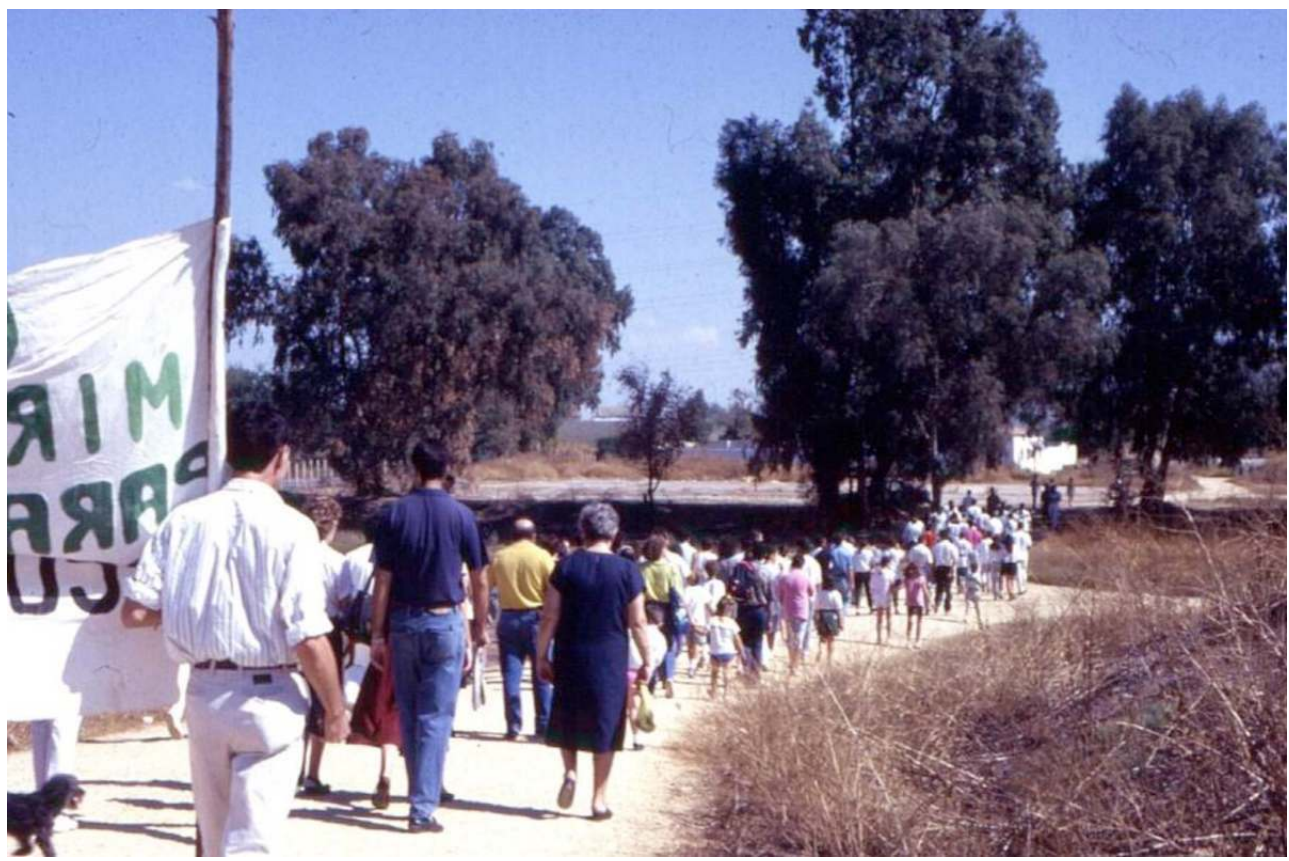

Source: Comité Pro-Parque Educativo Miraflores

Les Jardins du Parc de Miraflores se décomposent aujourd'hui en quatre projets complémentaires: "Les Jardins de loisir», "Les Jardins scolaires", "La Serre des jeunes » et les «Itinéraires pédagogiques ».

14 Les «Jardins de loisir » cherchent à développer diverses activités éducatives et ludiques. Pour ce faire, ils disposent de cent-quatre-vingts parcelles de 75 à $150 \mathrm{~m}^{2}$ d'agriculture horti-fruticole ou florale, exclusivement destinée à l'autoconsommation. À côté de ces parcelles individuelles a été réservée une zone d'espaces communs, où se trouvent des jardins et des arbres fruitiers entretenus par les jardiniers eux-mêmes ou des habitants des quartiers avoisinants. Les jardins de loisir sont destinés à des individus, des familles, associations et institutions publiques. Parmi ces individus et familles, on peut distinguer ceux qui participent en tant qu'horticulteurs ou jardiniers et ceux qui collaborent de manière désintéressée à quelques tâches collectives. Quant aux associations, elles travaillent pour la plupart avec des groupes et profils sociaux défavorisés, invalides physiques ou mentaux, schizophrènes, autistes, drogués en cure de désintoxication... L'organisation de ces jardins de loisir s'appuie sur les assemblées de jardiniers au cours 
desquelles sont prises les décisions collectives relatives à la résolution des problèmes identifiés par les usagers eux-mêmes : amélioration des infrastructures, activités à mener, cours, excursions, etc. Les jardiniers se réunissent par groupes, "chantiers » ou "ateliers", lorsqu'il s'agit de se lancer dans des travaux collectifs. Un chantier intitulé « compost » s'efforce par exemple tout à la fois d'apporter des solutions aux problèmes générés par les déchets végétaux et de sensibiliser les membres du collectif aux thèmes du recyclage, des engrais biologiques, etc.

Les «Jardins scolaires" sont pour leur part organisés par une coopérative de onze associations de parents d'élèves (AMPAs) des collèges publics des environs. Il s'agit d'une réponse à la faible, voire inexistante, implication des professeurs dans les activités du Jardin. Sauf cas exceptionnels, les enseignants rechignent en effet à se mobiliser en dehors des heures de travail si on ne leur offre pas de compensation économique. Parmi les activités réservées aux enfants, nous pouvons souligner l'importance des «jeux écologiques ", des ateliers éducatifs et du travail au jardin. L'expérience montre que ce dernier notamment constitue un excellent outil pédagogique, les enfants y apprenant à reconnaître les plantes, à pratiquer des semis, repiquer, arroser, récolter... L'activité se déroule sur l'ensemble de l'année scolaire, ce qui permet aux deux cent cinquante enfants impliqués de prendre conscience du cycle de vie de nombreuses plantes qu'ils ont l'habitude de voir chez eux (salades, tomates) sans, jusqu'ici bien connaitre leur provenance. Bien plus, ils s'habituent ainsi à une alimentation plus saine, en consommant plus volontiers des légumes et des produits qu'ils ont contribué à faire pousser.

La «Serre des jeunes » est quant à elle destinée à cinquante adolescents qui ont bénéficié l'année précédente des jardins scolaires et qui souhaitent poursuivre leur activité dans le parc. Ce sont eux qui se chargent de la serre et qui transmettent leurs savoirs et l'expérience acquise aux enfants des jardins scolaires.

Quant aux "Itinéraires pédagogiques", ils proposent des visites aux points les plus intéressants du Parc de Miraflores. Ils prétendent éveiller la conscience écologique du visiteur en lui faisant découvrir les curiosités environnementales, culturelles et historicoartistiques préservées dans le parc (vestiges archéologiques romains et arabes, moulin à huile du XVIII siècle, norias, etc.).

\section{Les autres expériences Sévillanes}

Parallèlement à l'expérience du Parc de Miraflores est née au cours des années 1980 la Confédération Écologiste Pacifiste d'Andalousie (CEPA), entité qui s'est transformée plus tard en Écologistes en Action. À la différence du modèle de Miraflores qui répond davantage à des préoccupations culturelles et éducatives, la CEPA crée en 1994 les jardins collectifs du Parc de San Jeronimo en mettant en avant des enjeux environnementaux et des pratiques culturales "alternatives ». Écologistes en Action ne vise pas à l'occupation d'espaces, mais entend arriver à des accords avec la mairie de Séville afin d'installer des jardins écologiques sur des terrains qui furent utilisés comme pépinières pour les plantes destinées à l'Exposition Universelle de 1992.

La troisième expérience de jardins collectifs à Séville se déroule dans le quartier du Parc Alcosa avec la création en 1996 de l'association Movida Pro-Parque du Tamarguillo dont les jardins urbains ont toutefois attendu 2006 pour réellement commencer à fonctionner. L'association reprend le modèle de Miraflores dans la mesure où les habitants du quartier 
ont "okupé » un terrain vide afin d'imposer un parc principalement composé par les jardins collectifs. Actuellement, deux zones horticoles sont en fonctionnement dans le Parc du Tamarguillo qui permettent à plus de deux cents familles de cultiver leur parcelle.

Viennent ensuite une série de création ou de « récupération » de jardins plus récents :

- En 2004, sera créé le Huerto del Rey Moro (Jardin du Roi Maure ). Il diffère des précédents dans la mesure où ceux-ci (Miraflores, San Jeronimo et Tamarguillo) exploitent des terrains en périphérie de la ville alors que le Jardin du Roi Maure se situe en plein centre historique de Séville. Il procède de la réunion de plusieurs mouvements sociaux locaux difficilement définissables, impliqués dans des actions revendicatrices (plutôt alternatives) propres au centre historique (Maison-Palais du Pumarejo, Centre social autogéré «Vieilles Demeures ", la Fabrique de Chapeaux, etc.). À ce jour, le jardinage se pratique dans des caissons ou des banquettes surélevées parce que le terrain a été déclaré « Bien d'intérêt culturel » et qu'il est interdit de le modifier. Le Huerto s'est lancé dans diverses expériences de permaculture, de concerts à l'air libre, de réunions politiques, etc.

- En 2007, seront créés les Jardins du quartier de Torreblanca. Contrairement aux précédents, ceux-ci ne résultent cependant pas d'une pratique revendicatrice, mais d'une proposition acceptée dans le cadre des Budgets participatifs de la Mairie. Ils se composent de vingt-trois parcelles de $75 \mathrm{~m}^{2}$ que gère l'association « Jardins familiaux de Torreblanca » montée par les bénéficiaires. Ils sont dépourvus de jardins scolaires car l'ensemble n'est destiné qu'aux adultes.

- En 2010, les Jardins de Pino Montano voient le jour. Ils s'installent à l'intérieur de l'ancien hôpital psychiatrique qui avait été désaffecté dans les années 1980. La Communauté générale des propriétaires de Pino Montano gestionnaire de l'ensemble utilise l'espace pour y développer d'autres activités. En plus de l'horticulture, elle a introduit des animaux dans le but de créer une ferme. De plus, six bungalows ont été construits qui peuvent être loués à des collèges ou à des associations désireux d'y passer des fins de semaine.

- En 2011, Les Jardins de l'Hacienda San Antonio ouvrent sur des terrains voisins de ceux de Torreblanca à l'initiative des membres de Torreblanca eux-mêmes, car ceux-ci avaient constaté que le nombre réduit (23) des parcelles existantes ne suffisait pas à satisfaire toutes les demandes émanant des habitants. Cinquante autres parcelles y sont mises en place en collaboration avec la Mairie.

- En 2012 les Jardins de Bellavista font leur apparition dans un autre des quartiers périphériques de la ville à la suite d'une pétition des habitants. Pour l'instant, 40 parcelles de $90 \mathrm{~m}^{2}$ sont dévolues aux adultes, sans qu'il soit fait mention de jardins scolaires.

- En 2011 toujours, les sérieuses carences et difficultés propres au quartier connu sous le nom de "Trois mille logements" ont entrâné la création des Jardins du Polygone Sud. Le quartier doit affronter de sérieux problèmes liés à la drogue, à l'insécurité urbaine et à un taux de chômage élevé. C'est cette situation qui a poussé les voisins à aménager vingt jardins à l'intérieur d'un collège afin que les familles les plus défavorisées puissent avoir accès à des aliments frais de qualité.

- En 2013, la Junta de Andalucía (le gouvernement régional de l'Andalousie) met en place le Parc de l'Alamillo, faisant de ces jardins les premiers à relever directement de l'administration publique. Le terrain est divisé en cent-trente-sept parcelles de 35 et $70 \mathrm{~m}^{2}$ destinées à des habitants de l'aire métropolitaine sévillane. 


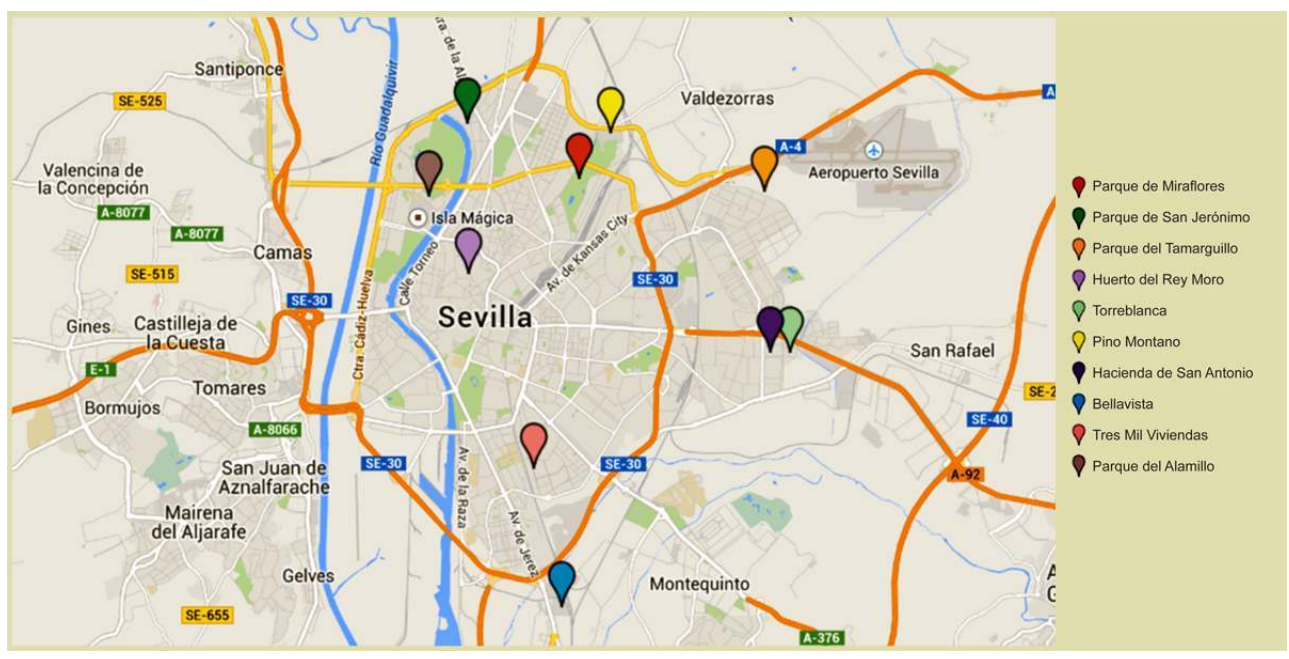

(C) R. PUENTE ASUERo

21 Il nous semble important de souligner certaines des similitudes qui caractérisent le paysage des jardins familiaux sévillans. En premier lieu, tous ces jardins répondent à la même finalité majeure, qui associe une activité de passe-temps à un désir d'autoconsommation, étant bien entendu que la vente de la production, légumes ou autres plantes potagères, y est interdite. En second lieu, le profil social majoritaire dans les jardins correspond à des hommes âgés et retraités. Alors que le jardin scolaire du parc Miraflores est le seul dans son genre, les femmes n'occupent qu'une place réduite et leur activité se limite le plus souvent à la culture des plantes ornementales. En troisième lieu, si la durée de l'occupation des parcelles est indéfinie, elle est partout sujette à des normes internes d'agriculture écologique et de bon voisinage. Le jardinier risque l'expulsion en cas de non-respect de ces normes. Tous les jardiniers affirment d'ailleurs pratiquer une agriculture "écologique». Toutefois, tous reconnaissent également qu'il leur est arrivé d'utiliser des produits phytosanitaires non-écologiques de façon sporadique. De la même manière, l'ensemble des jardins insistent sur leur gestion efficace de l'eau. Alors que s'était pratiqué pendant longtemps l'arrosage traditionnel par tuyaux, le recours à l'arrosage au goutte à goutte est de plus en plus présent. Finalement, le Jardin du roi maure est le seul à se présenter sous forme communautaire, c'est-à-dire que le terrain n'y est pas divisé en parcelles et que les membres du collectif cultivent tous le même espace. Dans les autres jardins, les parcelles attribuées ont une surface qui varie entre 50 et $150 \mathrm{~m}$ ${ }^{2}$. Chaque jardin s'auto-organise de manière indépendante en fonction de ce que décident les associations ou les gens du quartier. La municipalité de Séville n'a pas voté la moindre loi ou édicté de règlement susceptibles d'unifier les critères de gestion des collectifs.

\section{La non-reconnaissance par la Ville de la dynamique agri-urbaine}

Juan Espadas, le candidat du PSOE (Parti socialiste) à la mairie de Séville aux élections de 2011, avait proposé de créer un Réseau des Jardins Collectifs de Séville (RHUS). Ce réseau avait pour objectif d'installer un jardin collectif dans chaque district. Pour les plus étendus de ces districts, composés de plusieurs unités territoriales, l'idée consistait à 
disposer d'un jardin collectif par quartier. Cette proposition électorale ne s'est cependant pas concrétisée dans la mesure où le Parti socialiste a été battu lors des élections municipales. Il s'agissait là pourtant d'un signe fort de valorisation des dynamiques en cours qui, de fait, contraste fortement avec la position de la municipalité de Séville sur la question agri-urbaine.

Soulignons ainsi, qu'à l'exception de ceux de l'Alamillo, tous les jardins collectifs de Séville procèdent d'une initiative des habitants du quartier. Cette attitude citoyenne contraste évidemment avec la passivité dont fait preuve la mairie de Séville qui, alors même qu'elle compte sur son territoire quelques-uns des plus anciens jardins d'Espagne, continue de les tenir pour anecdotiques et de considérer les dynamiques présentées ici comme des expériences très minoritaires. Soulignons ainsi que seuls les jardins des parcs Miraflores, San Jerónimo et de l'Alamillo bénéficient de (faibles) subventions publiques. Les autres n'en disposent d'aucune. Plus encore, en dehors des conventions de collaboration signées avec Miraflores et San Jerónimo, la mairie ne sait que faire de ces jardins, s'interrogeant encore sur le district ou la délégation municipale qui devrait se charger de leur gestion. Elle n'a en tout cas pas voté le moindre budget prévisionnel, et il est paradoxal de constater que, alors que toutes les grandes villes du monde voient dans les jardins collectifs urbains une démarche particulièrement novatrice qu'il convient d'appuyer, Séville choisit de prendre une direction contraire.

\section{La situation de Séville dans le contexte andalou et espagnol}

L'existence de jardins collectifs en Andalousie était restée largement anecdotique jusqu'en 2008 (Figure 3) et elle se concentrait presqu'exclusivement sur Séville. Dans le contexte de crise actuel, et comme s'il s'agissait d'un mécanisme de réponse émanant directement des populations elles-mêmes, on assiste en Andalousie et en Espagne à un développement exponentiel de jardins familiaux et urbains qui ont une vocation évidente à la fois de garantie de souveraineté et de sécurité alimentaires, et d'assistance sociale pour des familles en plus ou moins grande difficulté. 
Figure 3 : Les jardins collectifs de l'Andalousie en 2008

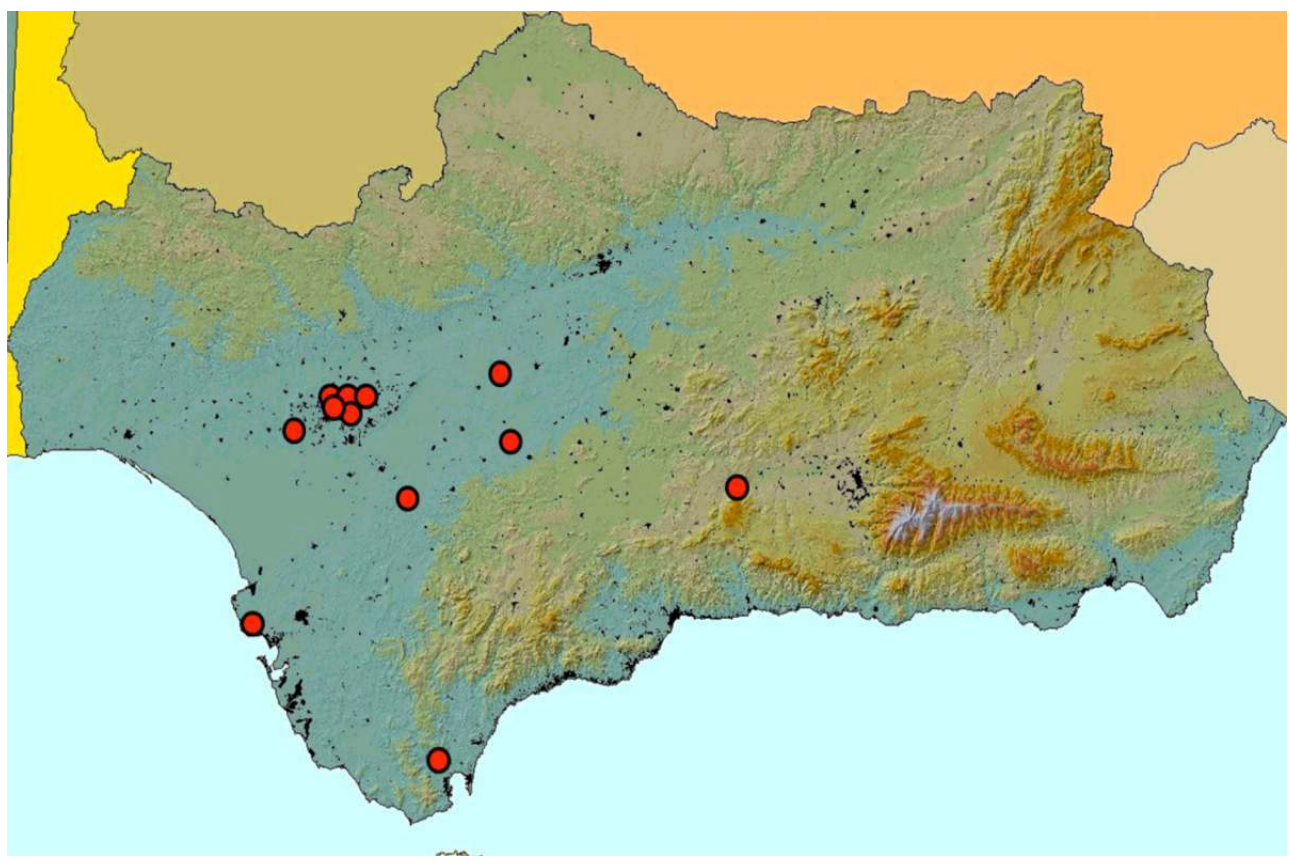

(C) R. Puente Asuero

Il s'est créé au cours des dernières années une centaine de jardins collectifs destinés à lutter contre la crise (Figure 4), démontrant une fois encore que l'agriculture urbaine ne se contente pas de fournir des produits de qualité, mais qu'elle offre aussi des espaces qui renforcent le tissu social dans les villes et les villages, agissant sous forme de mécanisme de résistance et de survivance face à des situations extrêmes comme le sont les crises économiques, les crises alimentaires, les conflits armés, etc. À leur propos, il n'est pas inutile de rappeler l'expression de Ron Finley, créateur des «guérillas urbaines de Los Angeles sud » : "faire pousser tes propres aliments, c'est comme imprimer tes propres billets ». 


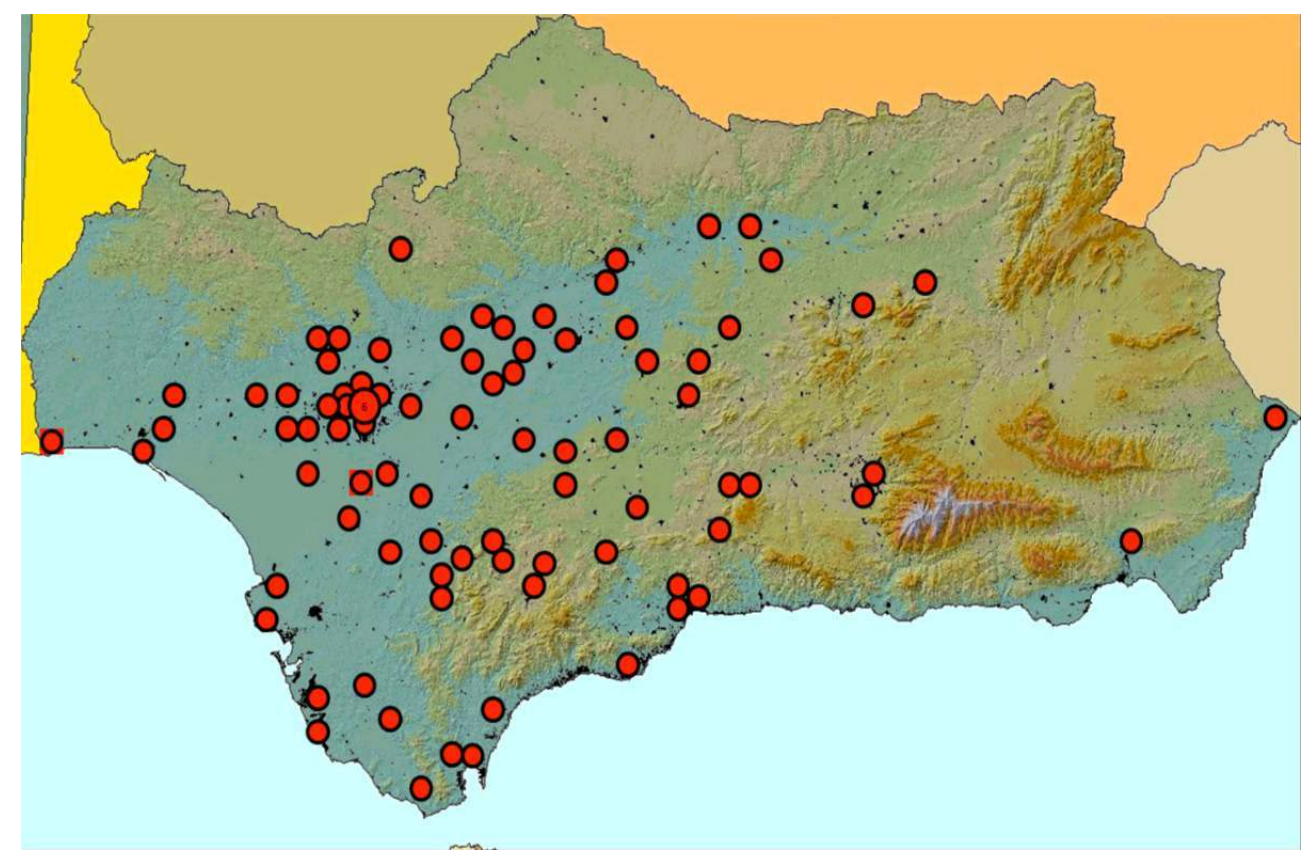

(c) R. Puente Asuero

On se doit de faire remarquer que les jardins se développent essentiellement dans les villes moyennes et grandes d'Andalousie alors que les agglomérations des régions montagneuses (au nord et à l'est) n'ont pratiquement pas ressenti le besoin de s'en doter. Cela démontre que de telles zones, où prédomine la petite propriété, parviennent à mieux résister à la crise économique. Dans la mesure où une plus grande part de la population y détient de petits terrains de culture, les municipalités n'ont guère de raison d'attribuer des parcelles collectives.

Il est pour l'heure encore difficile d'évaluer les effets positifs des jardins andalous compte tenu de leur courte existence et de l'absence de recherche systématique consacrée à la thématique. Certains indices permettent cependant de repérer une tendance au progrès dans quatre secteurs :

- social - Les jardins améliorent la qualité de vie des personnes grâce au développement des relations interpersonnelles et à l'exercice physique qu'ils procurent, contribuant ainsi à l'équilibre psychologique. De même, la production directe et immédiate de produits frais favorise-t-elle la consommation d'aliments de première nécessité, tels que salades, tomates, poivrons, aubergines, fèves... et elle permet en général de disposer d'un régime alimentaire plus sain ;

- économique - Les jardins permettent aux familles en réelle difficulté d'épargner. D'un autre côté, ils donnent l'occasion de relancer divers types de métiers annexes, comme la vente de graines, d'outils, d'engrais, le conseil professionnel, l'expertise en agriculture alternative, etc.;

- écologique - Les jardins contribuent à l'équilibre écologique des cycles naturels de l'eau et des sols, et à la diminution de l'effet de serre dans le climat urbain. Ils exercent également une action phytocide et antibiotique efficace contre la pollution microbienne en servant de filtres à air capables d'entraîner une diminution du nombre d'allergies et de maladies de type respiratoire ; 
- urbanistique - Les jardins permettent de revitaliser des espaces urbains sous-utilisés et/ou dégradés en se comportant souvent comme autant de zones tampon qui conduisent à mieux séparer les surfaces à usages incompatibles (hôpitaux, collèges situés en face de routes ou de bâtiments industriels).

C'est ainsi que si l'agriculture urbaine et périurbaine andalouse ne résoudra évidemment pas à elle seule les problèmes sociaux, économiques, territoriaux et écologiques qui accablent les villes, il semble acquis qu'elle contribuera très certainement à leur amélioration.

\section{Quelques réflexions en guise de conclusion}

Puisque Séville bénéficie d'une histoire longue des jardins sur son territoire, on pourrait penser que l'apparition de ces jardins collectifs ne représente qu'une simple évolution. Certains n'hésitent cependant pas à la qualifier de révolution en excipant du fait que ce sont des associations citoyennes qui s'imposent aujourd'hui comme les artisans de la restauration d'une activité, d'une mémoire et d'un paysage agricole ayant pratiquement disparu de la cité. Dans un tel contexte, on voit émerger à Séville, comme dans le reste de l'Espagne, des processus parallèles qui semblent parfois contradictoires.

Il y a tout d'abord une forme de contradiction dans l'apparition de nouveaux terrains agricoles destinés aux jardins sociaux dans des espaces dénués de «mémoire agricole » et en même temps la disparition à quelques mètres de là de parcelles horticoles traditionnelles situées sur les sols fertiles de la vallée d'un fleuve. Nos environnements urbains conservent de nombreuses surfaces horticoles que la logique demande de protéger et préserver puisqu'en règle générale il sera toujours préférable de les utiliser (quand c'est possible) plutôt que d'inventer des emplacements « inédits ».

31 Il y a ensuite une concomitance entre le développement de nouvelles formes d'agriculture de rente et d'une agriculture de loisir qui apparaissent plus en synergie qu'en concurrence. En effet, en même temps que se développent à rythme soutenu des jardins collectifs et familiaux principalement consacrés à l'autoconsommation et dépourvus de toute visée professionnelle ou commerciale, on assiste au retour d'une agriculture à caractère professionnel, tournée vers la vente, avec de nouveaux acteurs (citadins authentiques ou bohèmes urbains) et des formes de production et de commercialisation originales. L'ensemble nous apparaît trouver sa place de manière non concurrentielle dans une mouvance agro-écologique plus large qui est en train de se faire une place en Espagne à la suite de la crise et se caractérise tout à la fois par de nouveaux modes de production, de commercialisation et de consommation: intérêt croissant pour une production fondée sur des critères agro-écologiques, organisation de groupes d'autoproduction, magasins de produits de proximité, les produits « $\mathrm{km} 0 »$, restaurants écologiques, regroupements de consommateurs, etc. L'ensemble, qui traduit une réinvention des relations ville-campagne se nourrit d'une forme de confiance entre producteurs et consommateurs et de l'engagement des citoyens à consommer des produits en fonction de critères justes, éthiques, sociaux et écologiques.

32 En troisième lieu, il apparaît qu'à Séville et ailleurs, la création de jardins collectifs suppose des capacités d'intervention de la société civile lui permettant de récupérer ou de mettre en valeur des espaces urbains ou périurbains. Les jardins collectifs devraient ainsi se considérer comme des acupuncteurs urbains capables de soigner la ville sur les 
plans urbanistique et social. Plus encore, il n'est pas interdit de penser que les jardins écologiques sont en mesure de contribuer à l'émergence d'un nouveau modèle de la cité. (Fleury \& Vidal 2007).

Au moment où l'on cherche à valoriser la multifonctionnalité de l'espace urbain, et tandis qu'on ne cesse d'évoquer la crise des espaces publics, les jardins écologiques urbains s'imposent ainsi comme des espaces propres à renforcer le tissu social des quartiers environnants, parce qu'ils offrent un espace public vivant, chargé de contenu, symboliquement fort et qui le plus souvent se trouve à l'origine - à moins qu'il n'en soit la conséquence - de la conscience identitaire de ces quartiers. Mais l'idée de «faire la ville avec les jardins » ne doit pas déboucher sur les illusions bucoliques des citadins ou des bohèmes urbains vis-à-vis du monde rural et d'une agriculture susceptible de fabriquer des « espaces-spectacles » qui folkloriseraient l'activité agricole.

Finalement, même s'il existe à travers toute l'Espagne des centaines de jardins urbains, il manque toujours une loi régionale ou nationale qui protégerait ce type d'agriculture en ville. La reconnaissance institutionnelle dont bénéficient les jardins en Europe, avec des lois qui protègent et régulent, n'est pas encore parvenue en Espagne. La rédaction d'une loi andalouse ou espagnole sur le sujet constitue l'un des objectifs du futur proche. Il faudrait en tout cas que l'Espagne renforce les organisations étatiques et régionales de citoyens-agriculteurs qui s'inspireraient des modèles français de la Fédération Nationale des Jardins Familiaux et Collectifs, anglais de la National Allotmens Society ou allemand de la Bundesverband Deutscher Gartenfreunde. L'Espagne se trouve au tout début d'une tendance urbaine de niveau mondial. Les citoyens ont déjà pris l'initiative de constituer des groupes de production et de consommation, de construire des jardins collectifs, de former des coopératives et des entreprises d'économie sociale, c'est maintenant au tour des administrations de se tourner vers ce secteur et de s'investir en sa faveur de façon résolue.

\section{BIBLIOGRAPHIE}

Bejarano R., Puente R., Cáceres M. \& Porras C. 2000 - El Parque Miraflores de Sevilla, una isla verde con historia. In I Congreso Español de Biogeografía, Universitat de Girona.

Chassagne M. E. 1982 - Les effets de la planification spatiale sur les activités rurales. Revue Géographique des Pyrénées et du Sud-Ouest. 53 (1) : 51-66.

Fleury A. \& Vidal R. 2007 - Ville et agriculture périurbaine, de nouvelles formes territoriales. Revue Territoires, $474:$ 40-42.

García A., Oliveira G., Puente R. \& Torres F. J. 2007 - Ciudades en la ciudad. Mirando desde los espacios públicos de Sevilla. In Ambientes, ventanas y miradas en los paisajes de Andalucía y Marruecos. Sevilla, Consejería de Medio Ambiente.

Rodríguez López R. 2008 - El huerto en la Roma antigua: su problemática urbanística y agraria. Madrid, Ed. Dykinson. 


\section{NOTES}

1. Mot dérivé d' «occuper », utilisée par des groupes alternatifs, antisystème et avec une forte charge politique.

\section{RÉSUMÉS}

Le paysage traditionnel des jardins historiques de Séville s'est retrouvé remplacé au cours du xx siècle par un véritable «kaléïdoscope urbain " de zones résidentielles et d'infrastructures de transport. À partir de 1983, l'association Comité Pro-Parque Educativo Miraflores a décidé d'occuper un immense dépotoir de 90 hectares pour faire de cet espace dégradé un parc destiné à améliorer les conditions environnementales des quartiers périphériques de la ville. Trois décennies plus tard, on peut dire des voisins du Parc Miraflores qu'ils se sont fortement attelés à l'amélioration de la ville tout en revendiquant et en assumant la gestion de certains programmes sociaux. De nos jours, de nombreux jardins urbains se sont créés à Séville et en Espagne en s'inspirant du modèle du Parc Miraflores, certains par effet de mode, d'autres en réponse à la crise économique, mais tous constituent des espaces publics vivants qui renforcent le tissu social des agglomérations. De la même manière, les jardins urbains participent de la récupération de tout un pan des valeurs culturelles associées au milieu rural qu'on pensait oubliées. En dépit des aspects largement positifs de cette effervescence horticole, et comme elle en a l'habitude, l'administration publique (mairies, régions, État) a tardé à réagir et n'a pratiquement rien fait pour soutenir un mouvement qui vient de la rue.

El tradicional paisaje de huertas históricas de Sevilla se ha visto sustituido durante el s. XX por un auténtico "caleidoscopio urbano" de zonas residenciales e infraestructuras de transporte. A partir de 1983 la asociación Comité Pro-Parque Educativo Miraflores decidió ocupar una inmensa escombrera de 90 ha y transformar este solar degradado en un parque con la idea de mejorar estos barrios periféricos de la ciudad. Tras tres décadas, los vecinos/as del P. Miraflores se han "manchado" en la mejora de la ciudad reivindicando y asumiendo la gestión de programas sociales. Actualmente, son muchos los huertos urbanos en Sevilla y en España que han seguido el modelo del P. Miraflores, algunos como resultado de una moda, otros como respuesta a la crisis económica, pero todos son espacios públicos vivos que fortalecen el tejido social de las ciudades. Asimismo, los huertos urbanos están contribuyendo a recuperar unos valores culturales asociados al medio rural que parecían olvidados. Sin embargo, en este contexto de efervescencia hortelana, poco o muy poco ha hecho la administración pública (ayuntamientos, regiones y estado) que, una vez más, llega tarde al movimiento que viene desde la calle.

The traditional landscape of historical gardens of Seville has met replaced during the 20th century for an authentic "urban kaleidoscope" of residential zones and infrastructures of transport. From 1983 the association Comite Pro-Parque Educativo Miraflores decided to occupy an immense dump of 90 has and to transform this lot degraded in a park with the idea of improving these peripheral neighborhoods of the city. After three decades, the neighbors of the Miraflores Park have been involved actively in the improvement of the city claiming and 
assuming the management of social programs.

Nowadays, in the current context of multi-crisis and as mechanism of response of the population, there is taking place an exponential growth of the urban gardens in Spain with a welfare vocation, of self-consumption, of guarantor of the sovereignty and the food safety and as social resource for disadvantaged families.

Many urban gardens have been still the model initiated by the Miraflores Park and all has as common denominator that they are public alive spaces that strengthen the social fabric of the cities. Likewise, the urban gardens are helping to recover a few cultural values associated with the rural way that seemed to be forgotten.

Nevertheless, in this context of garden effervescence, little or very little there has done the public administration (city councils, regional government and national government) that, once again, is late to the movement that comes from the street.

\section{INDEX}

Index géographique : Séville, Espagne

Palabras claves : huertos urbanos, huertos familiares, crisis, movimientos sociales, patrimonio, multifuncionalidad

Mots-clés : jardins familiaux, crise, mouvements sociaux, patrimoine, multifonctionnalité

Keywords : urban gardens, familiar gardens, crisis, social movements, heritage, multifunctionality 